\title{
Exotic and Conventional Quarkonium Physics Prospects at Belle II
}

\section{Bryan Fulsom*†}

Pacific Northwest National Laboratory

E-mail: bryan. fulsomepnnl.gov

The Belle II experiment at the SuperKEKB energy-asymmetric $e^{+} e^{-}$collider is a substantial upgrade of the B factory facility at KEK in Tsukuba, Japan. It aims to record a factor of 50 times more data than its predecessor. The experiment completed a commissioning run in 2018, and began full operation in early 2019. Belle II is uniquely capable of studying the so-called "XYZ" particles: heavy exotic hadrons consisting of more than three quarks. First discovered by Belle, these now number in the dozens, and represent the emergence of a new category within quantum chromodynamics. This talk will present the prospects of Belle II to explore both exotic and conventional quarkonium physics.

XXIX International Symposium on Lepton Photon Interactions at High Energies - LeptonPhoton2019 August 5-10, 2019

Toronto, Canada

* Speaker.

${ }^{\dagger}$ On behalf of the Belle II Collaboration. 


\section{Exotic and Conventional Quarkonium Physics}

In the Standard Model of particle physics, hadronic matter is expected to consist of two- or three-quark combinations. One of the possible manifestations is quarkonium, the strongly-bound state of heavy quark-antiquark pairs $(c \bar{c}, b \bar{b})$. This system was first explained by phenomenological models, but effective field theories, and increasingly non-perturbative lattice calculations, now provide theoretical descriptions based on quantum chromodynamics (QCD), the Standard Model theory of the strong force interaction between quarks [1].

This area of research has recently experienced an incredible renaissance on the strength of numerous experimental results from the B-Factories and $e^{+} e^{-}$colliders. Discovery of the $X(3872)$ [2] was the first of a growing alphabet of quarkonium-like particles (i.e. $X(3872), Y_{c}(4260)$ [3], $Z_{c}^{ \pm}(3900)$ [4], and several of others [5]) that do not fit the well-established theoretical framework [1]. To date, the $X(3872)$ result remains the most highly-cited publication of the Belle Experiment [2]. Over the past decade, these newly discovered particles now number in the dozens, with most found in the charmonium sector accessible via $B$ decay and direct production. Analagous discoveries containing bottom quarks (e.g. $\Upsilon(5 S)$ decays to $Z_{b}^{ \pm}(10610 / 50)$ [6]) indicate that a similar family of particles may exist in the bottomonium sector.

Many of these discoveries are proposed to be exotic manifestations of QCD, including weaklybound meson molecules [7], tetraquarks [8], quark-gluon hybrids [9], and hadroquarkonium [10], or perhaps simply due to kinematic and threshold effects [11]. However, no satisfactory single comprehensive explanation has been found. Experiments indicate these many of these new states consist of four quarks, representing new forms of matter and quark binding in quantum chromodynamics. Further data are required to understand and disentangle the situation.

\section{Belle II Current Status}

The Belle II Experiment [12] is located at the SuperKEKB asymmetric $e^{+} e^{-}$collider at KEK in Tsukuba, Japan. This new experiment began with a commissioning run in 2018, and collected data with near-complete detector for $\sim 3$ months in 2019 [13]. The ultimate plan is to increase the luminosity (both instantaneous and integrated) by a factor of $\sim 40$ compared to the previous B-Factories, BaBar and Belle. In addition to the increased data set size, Belle II holds an advantage over hadron-collider experiments with decay modes involving neutrals and low-momentum particles, and the ability to fully reconstruct $B$ decay events. Particularly for studies of quarkonium, Belle II also has the ability to access multiple production mechanisms, including $B$ decays, initial state radiation (ISR), double-charmonium, $\gamma \gamma$ collisions, and by adjusting the beam energy to produce specific bottomonium states directly. While the nominal operation point is at the $\Upsilon(4 S)$ resonance, SuperKEKB has the potential to reach up to $11.24 \mathrm{GeV}$ in centre-of-mass energy for the production of bottomonium(-like) states.

In its very early data, Belle II has "rediscovered" several quarkonium states as a first step towards detector calibration, validation of tracking, particle identification and reconstruction ability, and physics analysis. Analyses have been performed on $\sim 2.62 \mathrm{fb}^{-1}$ of data collected at the $\Upsilon(4 S)$ resonance and, depending on the decay mode in question, an additional sample of $\sim 0.83 \mathrm{fb}^{-1}$ collected at an energy $60 \mathrm{MeV}$ below. At this stage, relatively straightforward selection criteria are 
applied to identify inclusively produced $J / \psi(1 S)$ decaying to both $e^{+} e^{-}$and $\mu^{+} \mu^{-}$final states. Calibration and efficiency of the Belle II electromagnetic calorimeter are probed by the former, while the dedicated $K_{L}^{0}$ and muon detector is tested by the latter. At this conference, observations of the $\psi(2 S)$ and $\Upsilon(1 S, 2 S, 3 S)$ states have been presented publicly for the first time by Belle II. $\psi(2 S)$ decays to $\mu^{+} \mu^{-}$are seen from two production methods: by extending the mass window of the inclusive $\mu^{+} \mu^{-}$search in $B$ meson decays that produced $J / \psi(1 S)$ signals, and by identifying $\psi(2 S) \rightarrow \pi^{+} \pi^{-} J / \psi(1 S)$ decays in few-track ISR events. The former use the $2.62 \mathrm{fb}^{-1}$ data sample, while the latter include the off-peak data for a total of $3.45 \mathrm{fb}^{-1}$. The $\psi(2 S)$ results are shown in Fig. 1. A similar technique is employed to isolate $\Upsilon(3 S) \rightarrow \pi^{+} \pi^{-} \Upsilon(1 S)\left(\mu^{+} \mu^{-}\right)$ and $\Upsilon(2 S) \rightarrow \pi^{+} \pi^{-} \Upsilon(1 S)\left(\mu^{+} \mu^{-}\right)$decays (for which the ISR production mechanism is completely dominant). The observed cross sections for $\Upsilon(2 S, 3 S)$ production are consistent with expectations. The $\Upsilon$-related results are shown in Fig. 2.
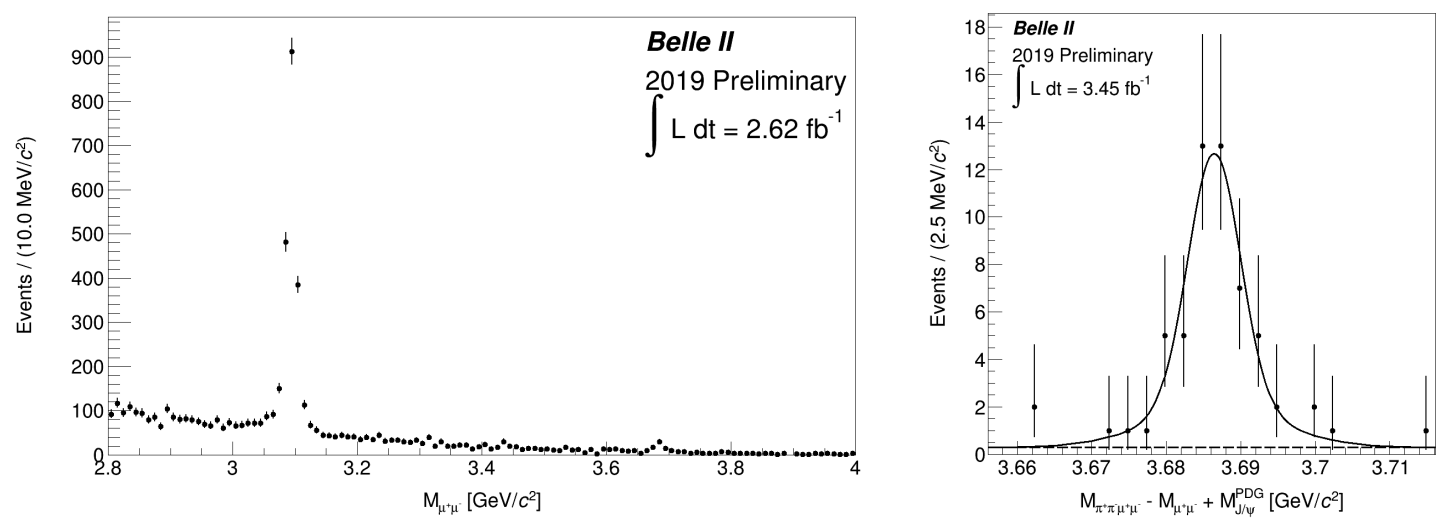

Figure 1: (Left) Inclusive dimuon events preferentially selected from $B$ meson decays. The visible peaks indicate $J / \psi(1 S)$ and $\psi(2 S)$ signals. (Right) Observation of $\psi(2 S)$ from ISR decaying via $\psi(2 S) \rightarrow$ $\pi^{+} \pi^{-} J / \psi(1 S)$. To improve resolution, the difference between the reconstructed $\psi(2 S)$ and $J / \psi(1 S)$ invariant masses is used in the plot.

\section{Belle II Future Prospects}

The Belle II experiment is expected to operate over the next decade, aiming to collect $50 \mathrm{ab}^{-1}$ of data to accomplish a wide-ranging physics program including searches for physics beyond the Standard Model in $B$ meson, charm, and $\tau$ decays, and in the dark sector. In addition, an extensive quarkonium physics program is planned to study both conventional and exotic states. Here we focus on a few specific items; for complete details of the Belle II physics program we refer the reader elsewhere [14].

Despite over 15 years of reseach, the nature of the $X(3872)$ is still unknown. With its large data set, Belle II should be able to definitively measure the branching fraction of $B^{ \pm} \rightarrow K^{ \pm} X(3872)$ based on the $K$ recoil technique [15]. Measurements of the natural width of the $X(3872)$, currently limited to $<1.3 \mathrm{MeV}$, will also be improved upon. Using $D^{0} \bar{D}^{0} \pi^{0}$ decays in the full dataset, a measurement of $5 \sigma$ significance can be made down to a width of $570 \mathrm{keV}$, or $90 \%$ confidence level limit set as low as $180 \mathrm{keV}$. 


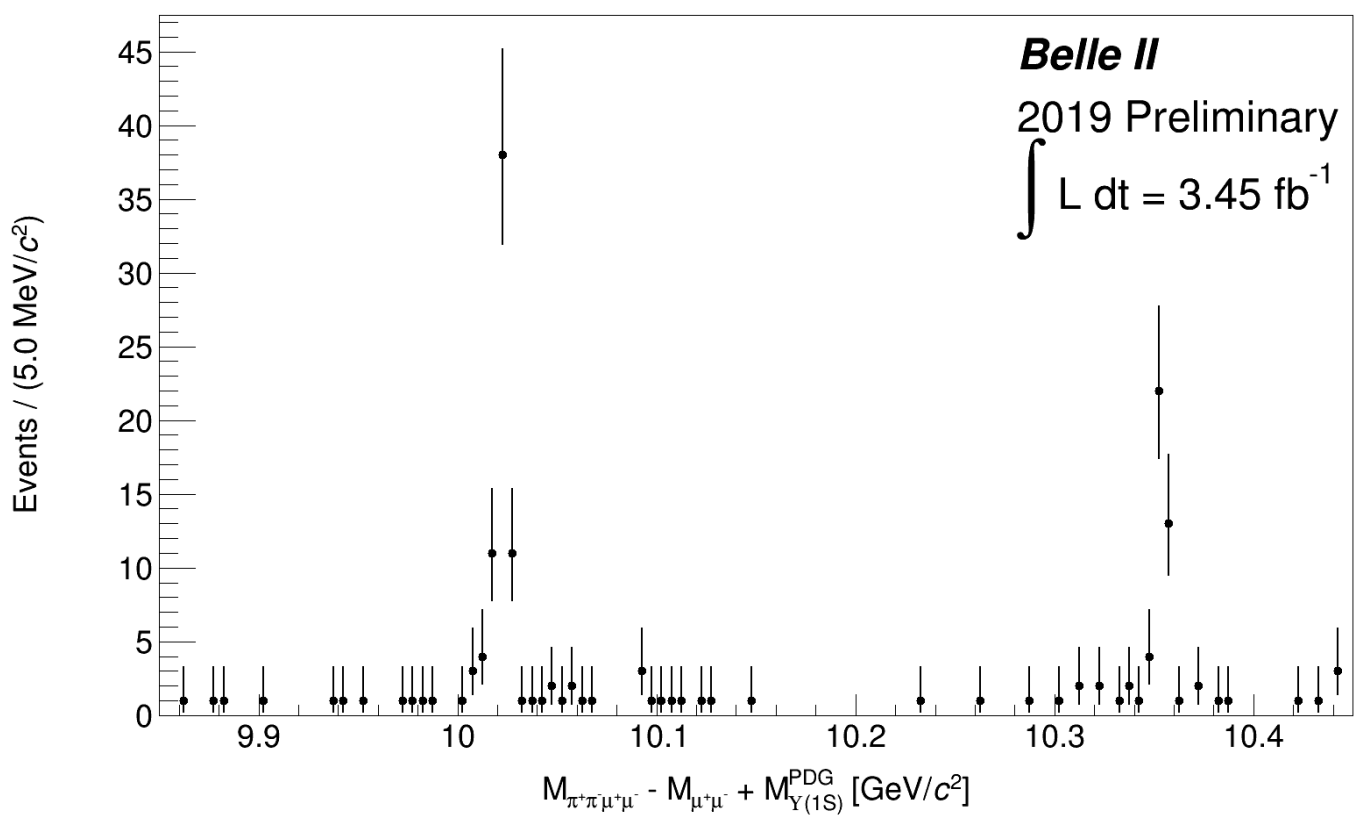

Figure 2: Observation of $\Upsilon(2 S)$ and $\Upsilon(3 S)$ decaying to $\pi^{+} \pi^{-} \Upsilon(1 S)\left(\mu^{+} \mu^{-}\right)$. To improve resolution, the difference between the reconstructed $\Upsilon(2 S, 3 S)$ and $\Upsilon(1 S)$ invariant masses is used in the plot.

ISR production of charmonium and exotic states, as used to provide the first indications of $\psi(2 S)$ and $\Upsilon$ at Belle II presented in this talk, can be exploited to cover a wide mass range. This is in contrast with, e.g., the BESIII experiment operating at specific centre-of-mass energies. At 10 (50) $\mathrm{ab}^{-1}$ Belle II is estimated to have an equivalent on-peak luminosity between $300-500(1500-2750) \mathrm{pb}^{-1} / 10 \mathrm{MeV}$ over a range of $3-5 \mathrm{GeV}$. Double-charmonium production of $J / \psi(1 S)$ with an accompanying charmonium(-like) state [16] will be improved by increased statistics, and searches for new states recoiling against charmonium of other quantum numbers (e.g. $\eta_{c}$ and $\chi_{c J}$ ) become a possibility. Similarly, two-photon interactions with greater statistics can be used to probe exotics such as putative four-quark $c \bar{c} s \bar{s}$ states decaying to $\phi J / \psi(1 S)$ [17].

Finally, Belle II and SuperKEKB have the unique opporunity to explore bottomonium(-like) states by scanning over or operating at centre-of-mass energies near the $\Upsilon(4 S)$ resonance. Only a relatively small amount of data have been collected at non- $\Upsilon(4 S)$ energies: $\mathscr{O}(10) \mathrm{fb}^{-1}$ at $\Upsilon(1 S, 2 S$, $3 S, 6 S), \mathscr{O}(100) \mathrm{fb}^{-1}$ at $\Upsilon(5 S)$, and typically $<1 \mathrm{fb}^{-1}$ at intermediate points. With the upgraded luminosity expected at SuperKEKB, a limited amount of time would be required to collect non- $\Upsilon(4 S)$ samples an order of magnitude larger. Efforts by previous experiments spanning the range from $\Upsilon(1 S)$ up to $\Upsilon(6 S)(\sim 11 \mathrm{GeV})$ led to the discovery of several bottomonia $\left(\eta_{b}(1 S, 2 S), h_{b}(1 P, 2 P)\right.$, and $\left.\Upsilon\left(1 D_{2}\right)\right)$, and possible four-quark states $\left(Z_{b}^{ \pm}(10610,10650)\right)$. In particular, there are recent hints from Belle $[18,19]$ for $Y_{b}$ states analgous to those in the charm sector. These particles can only be understood with increased statistics and a detailed scan with more energy points. Operating points below the $\Upsilon(4 S)$ open the possibilty to test predictions of physics beyond the Standard Model in $\Upsilon$ decays to invisible or lepton flavour violating final states. 


\section{Summary}

Interest in the quarkonium system has been revitalized by discoveries from the $B$-Factory era, and we are still searching for explanations of families of exotic multiquark particles. The Belle II experiment is the next generation $B$-Factory experiment and has recently begun data collection, providing the first indications of expected charmonium and bottomonium states. It offers unique opportunities to study quarkonium(-like) particles via a multitude of production and decay mechanisms, and is poised to continue the recent successes in this area over the years to come.

\section{References}

[1] For comprehensive modern reviews see N. Brambilla et al., CERN-2005-005 (2005), and N. Brambilla et al., Eur. Phys. J. C 71, 1 (2010).

[2] S.-K. Choi et al. (Belle Collaboration), Phys. Rev. Lett. 91, 262001 (2003).

[3] B. Aubert et al. (BaBar Collaboration), Phys. Rev. Lett. 95, 142001 (2005).

[4] M. Ablikim et al. (BESIII Collaboration), Phys. Rev. Lett. 110, 252001 (2013).

[5] Summarized in "Developments in heavy quarkonium spectroscopy" in K.A. Olive et al. (Particle Data Group), Chin. Phys. C 38, 090001 (2014).

[6] A. Bondar et al. (Belle Collaboration), Phys. Rev. Lett. 108, 122001 (2012).

[7] N.A. Tornqvist, Phys. Lett. B 590, 209 (2004).

[8] L. Maiani et al., Phys. Rev. D 89, 114010 (2014).

[9] T. Barnes et al., Phys. Rev. D 52, 5242 (1995).

[10] S. Dubynskiy et al., Phys. Lett. B 671, 82 (2009).

[11] E.S. Swanson, Phys. Rev. D 91, 034009 (2015).

[12] T. Abe, arXiv:1011.0352 (2010).

[13] For a recent general update from Belle II, see the talk by T. Browder at this conference, to appear in PoS(LeptonPhoton2019).

[14] E. Kou et al., arXiv:1808.10567 (2018).

[15] Y. Kato et al. (Belle Collaboration), Phys. Rev. D 97, 012005 (2018).

[16] P. Pakhlov et al. (Belle Collaboration), Phys. Rev. D 79, 071101 (2009).

[17] C.P. Shen et al. (Belle Collaboration), Phys. Rev. Lett. 104, 112004 (2010).

[18] R. Mizuk et al. (Belle Collaboration), Phys. Rev. Lett. 117, 142001 (2016).

[19] R. Mizuk et al. (Belle Collaboration), arXiv:1905.05521 (2019). 